\title{
Do young Suns undergo magnetic reversals?
}

\author{
Stephen C. Marsden ${ }^{1}$, Sandra V. Jeffers ${ }^{2}$, Jean-Francois Donati ${ }^{3}$, \\ Matthew W. Mengel ${ }^{4}$, Ian A. Waite ${ }^{4}$ and Brad D. Carter ${ }^{4}$ \\ ${ }^{1}$ Anglo-Australian Observatory, P.O. Box 296, Epping, NSW 1710, Australia \\ email: scm@aao.gov.au \\ ${ }^{2}$ Sterrekundig Instituut, Universiteit Utrecht, P.O. Box 80000, NL-3508 TA Utrecht, The \\ Netherlands \\ email: s.v.jeffers@uu.nl \\ ${ }^{3}$ Laboratoire d'Astrophysique de Toulouse-Tarbes, Observatoire Midi-Pyrénées, 14 avenue \\ Edouard Belin, F-31400 Toulouse, France \\ email: donati@ast.obs-mip.fr \\ ${ }^{4}$ Faculty of Sciences, University of Southern Queensland, Toowoomba 4350, Australia \\ email: mengel@usq.edu.au, waite@usq.edu.au, carterb@usq.edu.au
}

\begin{abstract}
A key part of the modern-day regenerative solar magnetic dynamo is the reversal of the Sun's global magnetic field every eleven years. However, recent theoretical models indicate that young-rapidly rotating Sun-like stars may not always undergo full magnetic reversals, but instead may sometimes undergo "attempted" reversals where the magnetic field declines in strength only to return with the same polarity. Using the technique of Zeeman Doppler imaging we have mapped the magnetic field topology of a small sample of young Sun-like stars at multiple epochs, and present tentative evidence of an "attempted" magnetic field reversal on one of our stars.
\end{abstract}

Keywords. stars: late-type, stars: imaging, stars: magnetic fields

\section{Introduction}

The generation of magnetic fields is arguably one of the most important process operating inside a star, influencing everything from the angular momentum evolution of the star through to the habitibility of planets. On the Sun, the reversal of the magnetic field every 11 years is a crucial element of the cyclic solar dynamo, however, what of young solar-type stars? Such stars are often rapidly-rotating and show activity phenomena, such as spots and flares, an order of magnitude or more larger than that of today's Sun, indicating powerful magnetic dynamos. Do such stars have a similar dynamo operation to that of the modern-day Sun or are they fundamentally different? Determining if young Sun-like stars have magnetic cycles is an important step in our understanding of how they produce magnetic fields.

Photometry of young solar-type stars as well as emission in the Calcium $\mathrm{H}$ and $\mathrm{K}$ lines from such stars, show variations and sometimes cycles in their emission level (i.e. Lanza 2009 and Baliunas et al. 1995). While the photometry of young solar-type stars often shows cyclic variations, the variations in the Calcium $\mathrm{H}$ and $\mathrm{K}$ flux from such young stars appears to be for the most part chaotic or irregular (Baliunas et al. 1995). Do these cycles/variations involve reversals of the magnetic field as in the solar case? To determine this we need to be able to observe and monitor the evolution of magnetic fields on young solar-type stars. 


\section{Detecting and mapping magnetic fields}

We can use the fact that starlight will be polarised in the presence of stellar magnetic fields in order to detect magnetic fields on the surfaces of stars. High-resolution spectropolarimeters, such as ESPaDOnS (on the Canada-France-Hawaii Telescope), NARVAL (on the Télescope Bernard Lyot) and SEMPOL (on the Anglo-Australian Telescope) split the starlight into right- and left-handed circularly polarised light. A Stokes V profile can then be reconstructed from the polarised light to reveal the presence of magnetic fields on the stellar surface.

Zeeman signatures in cool stars are usually extremely small, often with amplitudes $<0.1 \%$ of the continuum level. Thus spectra with signal-to-noise $>1000$ are required to even detect these signatures. Unfortunately, we usually cannot simply extend the exposure time in order to increase the signal-to-noise as the young stars we wish to observe are often rapidly-rotating (some with rotational periods of a fraction of a day). If the exposure time is increased too much the magnetic signatures will be smeared out as the star rotates during the exposure. However, when observing with an echelle, each spectrum contains several thousand photospheric lines which are all affected by the presence of surface magnetic fields in a similar way. Thus we can use the technique of Least-Squares Deconvolution (LSD, Donati et al. 1997) to combine these lines into one high signal-to-noise LSD profile. Using LSD can provide a multiple gain of $30+$ in signal-to-noise over that of a single line.

This allows us to detect the presence of magnetic fields on the surfaces of young solartype stars, but in order to determine if such stars are undergoing magnetic reversals we need to be able to map the surface magnetic topology of such stars. Magnetic regions on the stellar surface cause distortions in the star's Stokes V LSD profile which travel through the profile as the star rotates. Radial magnetic field (field in/out of the stellar surface) will produce a Stokes $\mathrm{V}$ profile that retains its sign as it passes across the stellar disk. In contrast, a region of azimuthal field (field wrapped around the star's rotation axis) will produce a Stokes $\mathrm{V}$ profile that changes sign as it passes across the stellar disc. Thus the orientation of the field can be determined. The longitude of a magnetic region is easily determined by the timing of the region passing through the centre of the profile. The latitude of a magnetic region on the stellar surface can also be determined as those regions near the equator will pass through the entire LSD profile while those regions nearer the pole will be restricted to the central part of the profile. Thus the position and orientation of magnetic regions on the stellar surface can be determined.

A time series of Stokes V LSD profiles taken over the stellar rotation period (or a significant part of it) is inverted using Zeeman Doppler imaging (ZDI, Brown et al. 1991, Donati \& Brown 1997) to reproduce the magnetic topology of the star. Unfortunately, this inversion is an ill-posed problem with an infinite number of solutions that can be found to fit the data to the noise level. In order to choose a unique solution some additional constraint, called regularisation, is usually applied. Our code uses the Skilling \& Bryan (1984) maximum entropy optimisation scheme which produces an image with the minimum amount of information required to fit the data.

\section{The evolution of magnetic topologies}

The technique of ZDI has been around for a number of years now and applied to a number of stars. So has a magnetic reversal actually been seen? Donati et al. (2003a) studied the magnetic topologies of several K-stars over a 7-11 year period and did not find any evidence for a magnetic reversal. However, all these stars have low levels of 
differential rotation (Donati et al. 2003b) and this may mean they have long magnetic cycles (see Section 3.2).

The only stars (outside of the Sun) so far to have evidenced a reversal of their magnetic field are the late-F star Tau Boo (Donati et al. 2008, Farès et al. 2009) and the Sun-like star HD 190771 (Petit et al. 2009). Tau Boo has shown two reversals in the polarity of its magnetic field in the space of two years leading to the belief that this star has a cycle length of $\sim 2$ years. This is significantly shorter than that of the Sun $(\sim 22$ years). What could be causing this? There are several possibilities. Firstly, Tau Boo has an extremely high level of surface differential rotation at almost 10 times the solar value and Donati et al. (2008) suggest that such a high level of differential rotation could well be responsible for the rapid magnetic cycle of the star. However, Tau Boo is also the host of a "Hot Jupiter" with a period of a few days (Butler et al. 1997) and Farès et al. (2009) suggest that this could be responsible.

\subsection{Young solar-type stars}

Both Tau Boo and HD 190771 are older than 1 Gyr and may well already have started to develop magnetic cycles similar to the Sun (although possibly much more rapid), but what of younger solar-type stars? Do such active, rapidly-rotating stars show evidence of magnetic reversals?

HD 171488 is a young (30 - 50 Myr old) early-G star which has had its magnetic topology mapped at four epochs from September 2004 through to November 2007 (see Figure 1). The most striking feature on the star is the ring of azimuthal magnetic field around the pole which is seen in all epochs. There appears to be little evidence of a reversal of the star's magnetic field over the 3 year period.

HR 1817 is a young (10 - 30 Myr old) late-F star which has had its magnetic topology determined at three epochs, December 2001, December 2007, and December 2008 (see Figure 2). A polar ring of azimuthal field is also in evidence at all three epochs, however, in 2007 the ring is significantly weaker and increased regions of negative azimuthal field are seen on the lower-latitudes of the star at this epoch. Again there is little evidence of a reversal of the magnetic field on the star.

\subsection{The role of differential rotation}

If we observe a star over several rotations the magnetic features will evolve under the influence of differential rotation. A simplified solar-like differential rotation law can be included in the imaging process:

$$
\Omega(l)=\Omega_{\mathrm{eq}}-d \Omega \sin ^{2} l,
$$

where $\Omega_{\mathrm{eq}}$ is the equatorial rotation rate and $d \Omega$ is the shear from equator to pole (the differential rotation). By treating $\Omega_{\mathrm{eq}}$ and $d \Omega$ as free parameters we can determine the differential rotation on a star using the $\chi^{2}$ minimisation method of Petit et al. (2002).

This has been done for both HD 171488 and HR 1817 (Marsden et al. 2006, Jeffers \& Donati 2008, Marsden et al. in prep.) and both have been shown to have extremely high levels of differential rotation, similar to that evidenced by Tau Boo at up to 10 times the solar value. In contrast to Tau Boo, neither star shows any evidence of a magnetic reversal over a 3 year period for HD 171488 and a 7 year period for HR 1817, although the long gap in observations between 2001 and 2007 could well mean that a magnetic reversal (or two) may well have been missed on HR 1817. Does this mean that differential rotation does not play a strong role in the length of a star's magnetic cycle? Does it mean that young solar-type stars do not in fact undergo magnetic reversals or are other factors at work? 
Radial field (September 2004)

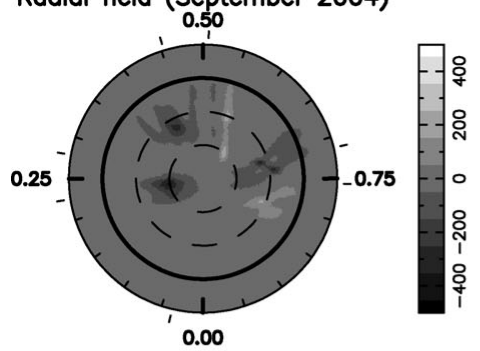

Radial field (June 2005)

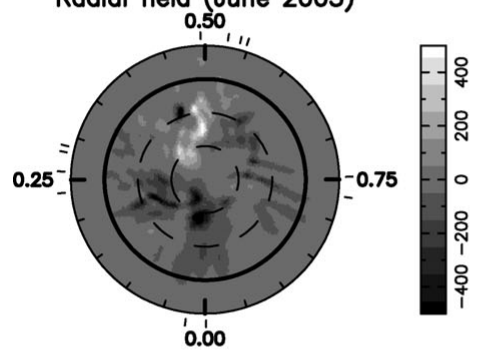

Radial field (May 2007)

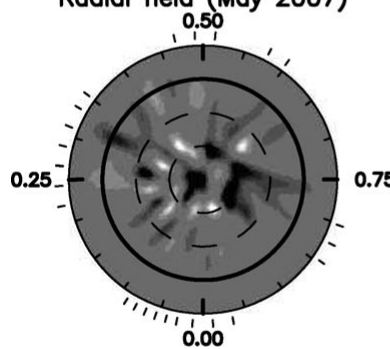

Radial field (November 2007)

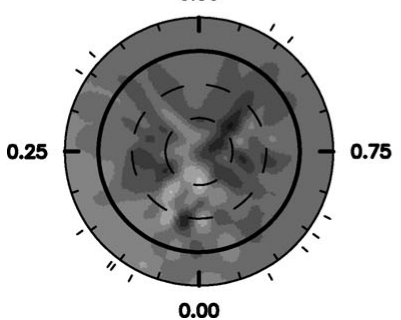

Azimuthal field (September 2004)
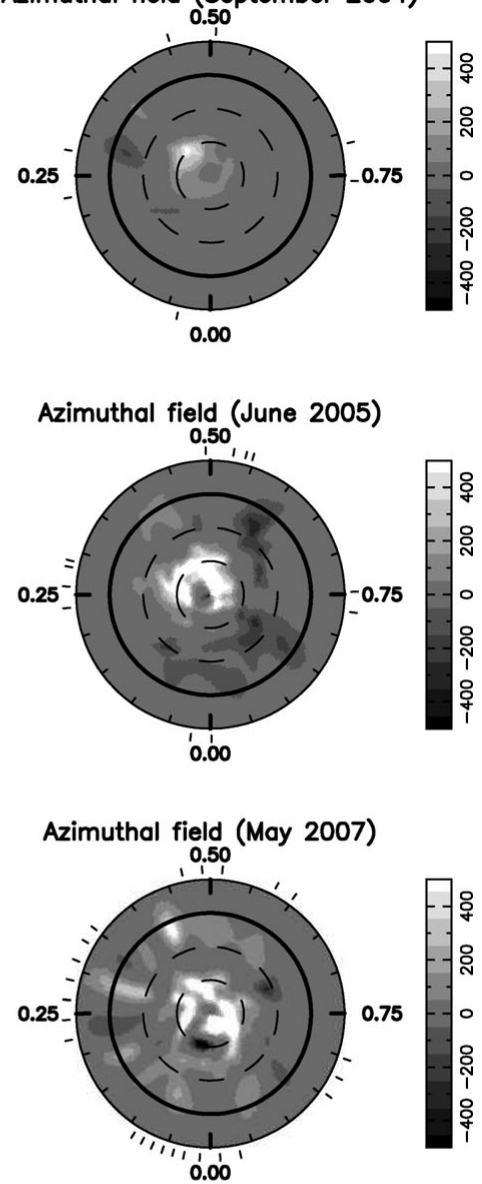

Azimuthal field (November 2007)

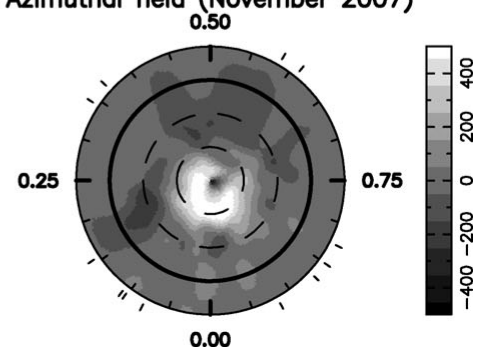

Figure 1. Radial (left) and azimuthal (right) magnetic topologies for HD 171488. The images are flattened polar projections extending down to $-30^{\circ}$ latitude. The bold line denotes the equator and the dashed lines are $+30^{\circ}$ and $+60^{\circ}$ latitude parallels. The radial ticks outside the plots indicate the rotational phases at which the star was observed. The magnetic scale is in gauss (Marsden et al. 2006, Jeffers \& Donati 2008, Jeffers et al. in prep.).

\section{Comparison with theory}

Recent theoretical models of rapidly-rotating (up to 5 times solar) young Suns by Brown et al. (2009) show that the dynamo of such stars appears to be distributed throughout the convection zone (rather than restricted to the interface layer as in the 

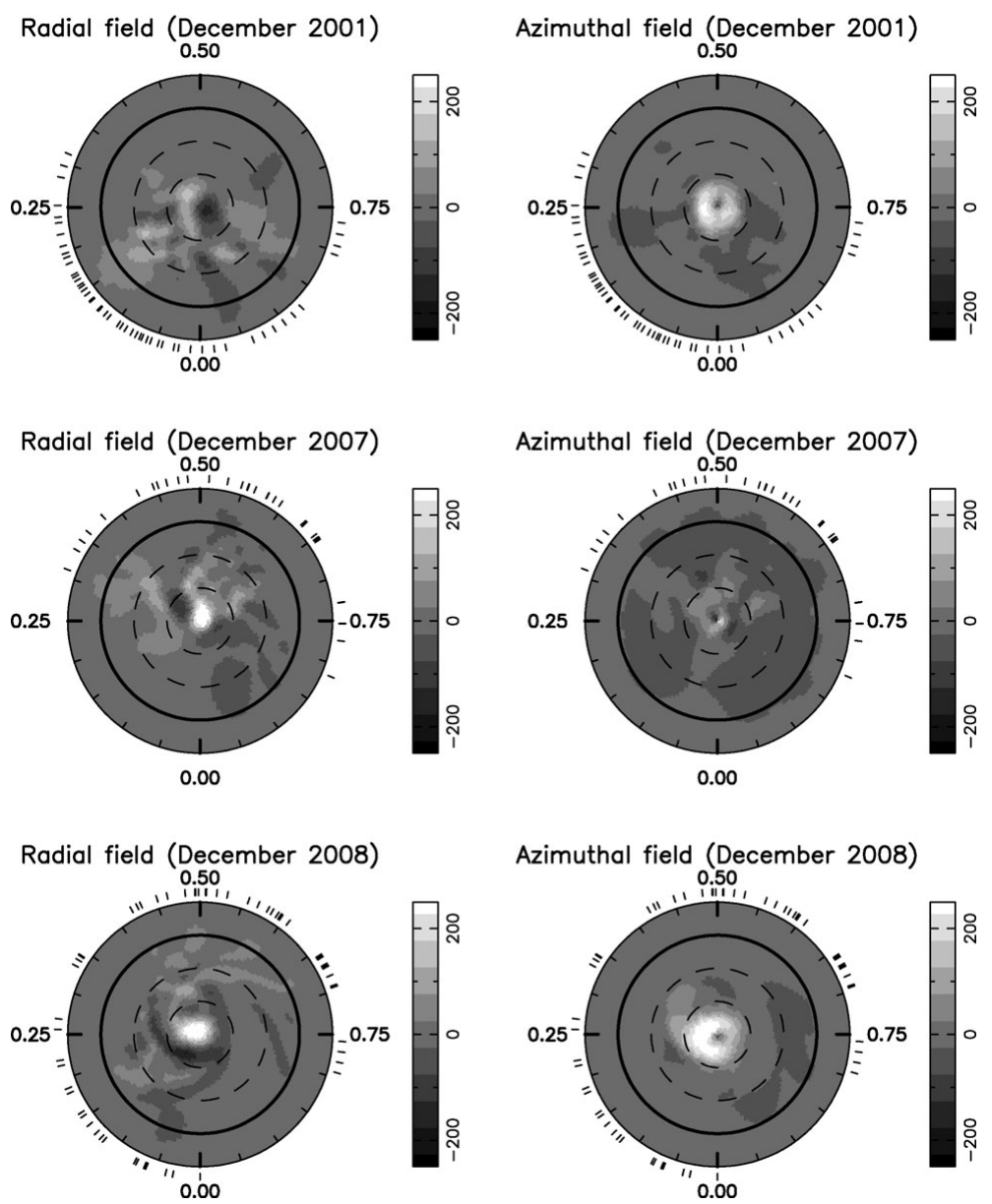

Figure 2. Radial (left) and azimuthal (right) magnetic topologies for HR 1817. The images are as in Figure 1 (Marsden et al. in prep.).

solar case). This appears to support the finding of large regions of near-surface azimuthal field on young solar-type stars which should not appear if a solar-type interface-layer dynamo is in operation. Futhermore the models of Brown et al. (2009) show that young Suns produce "wreaths" of azimuthal magnetic field near the stellar surface. These "wreaths" appear to be somewhat similar to those shown in our magnetic topologies (Figures 1 and 2) but at lower latitudes than those we see. Could this be due to the fact that our stars are much more rapidly rotating ( $\sim 20$ times solar) than the models of Brown et al. (2009), with the Coriolis force deflecting the wreaths to higher latitudes?

In addition, the models of Brown et al. (2009) show another interesting aspect. They show that not only do rapidly rotating Suns undergo full magnetic reversals but also undergo a number of "attempted" reversals, where the magnetic field strength in both the Northern and Southern hemispheres decreases but then strengthens with the same polarity rather than undergoing a reversal. Could this be what happened on HR 1817 in December 2007 with the weakening of its polar azimuthal ring?

\section{Conclusions}

We have used Zeeman Doppler imaging to reconstruct the magnetic field topology of two young solar-type stars at multiple epochs. Both stars show an intense ring of 
azimuthal field around the poles at almost all epochs with little evidence of a magnetic reversal. Such rings of azimuthal field are seen on a number of stars and appear similar to the "wreaths" of magnetic field produced by the models of Brown et al. (2009) except they are at higher latitudes, perhaps due to higher rotation rates. The young late-F star HR 1817 shows a significant weakening of its polar azimuthal ring during one epoch. We postulate that HR 1817 may have undergone an "attempted" reversal, as predicted by the models of Brown et al. (2009), during this epoch.

Only one solar-type star (outside of the Sun) has shown a possible magnetic cycle (Tau Boo) with a cycle length an order of magnitude shorter than that of the Sun. Tau Boo has both a high level of differential rotation and hosts a "Hot Jupiter", either of which may be influencing the magnetic cycle of the star. However, both of the young Sun-like stars we have studied (HD 171488 and HR 1817) have similarly high levels of differential rotation, but do not show any evidence of magnetic reversals, even though the observations cover a period similar to the cycle length of Tau Boo. Does this imply that the "Hot Jupiter" of Tau Boo, rather than high differential rotation, is somehow influencing its magnetic cycle? If so, how? Does the fact that both the stars that have shown magnetic reversals are older than $\sim 1$ Gyr mean that solar-type stars have developed regular magnetic cycles by this age?

So, do young Suns undergo magnetic reversals? The jury is still out. Theory says they should, along with undergoing "attempted" reversals, but it will take a dedicated observing program to monitor such stars in order to determine if they do and if so, how often and regularly they do.

\section{References}

Baliunas, S. L., Donahue, R. A., Soon, W. H., Horne, J. H., Frazer, J., Woodard-Eklund, L., Bradford, M., Rao, L. M., Wilson, O. C., Zhang, Q., Bennett, W., Briggs, J., Carroll, S. M., Duncan, D. K., Figueroa, D., Lanning, H. H., Misch, A., Mueller, J., Noyes, R. W., Poppe, D., Porter, A. C., Robinson, C. R., Russell, J., Shelton, J. C., Soyumer, T., Vaughan, A. H., \& Whitney, J. H. 1995, ApJ, 438, 269

Brown, S. F., Donati, J.-F., Rees, D. E., \& Semel, M. 1991, A\&广A, 250, 463

Brown, B. P., Browning, M. K., Brun, A. S., Miesch, M. S., \& Toorme, J. 2009, ApJ, submitted (arXiv:0906.2407)

Butler, R. P., Marcy, G. W., Williams, E., Hauser, H., \& Shirts, P. 1997, ApJ, 474, L115

Donati, J.-F. \& Brown, S. F. 1997, A\& A, 326, 1135

Donati, J.-F., Semel, M., Carter, B. D., Rees, D. E., \& Cameron, A. C. 1997, MNRAS, 291, 658

Donati, J.-F., Collier Cameron, A., Semel, M., Hussain, G. A. J., Petit, P., Carter, B. D., Marsden, S. C., Mengel, M., López Ariste, A., Jeffers, S. V., \& Rees, D. E. 2003a, MNRAS, 345,1145

Donati, J.-F., Collier Cameron, A., \& Petit, P. 2003b, MNRAS, 345, 1187

Donati, J.-F., Moutou, C., Farès, Bohlender, D., Catala, C., Deleuil, M., Shkolnik, E., Cameron, A. C., Jardine, M. M., \& Walker, G. A. H. 2008, MNRAS, 385, 1179

Farès, R., Donati, J.-F., Moutou, C., Bohlender, D., Catala, C., Deleuil, M., Shkolnik, E., Cameron, A. C., Jardine, M. M., \& Walker, G. A. H. 2009, MNRAS, 398, 1383

Jeffers, S. V. \& Donati, J.-F. 2008, MNRAS, 390, 635

Lanza, A. F. 2009, these proceedings, p. 120

Marsden, S. C., Donati, J.-F., Semel, M., Petit, P., \& Carter, B. D. 2006, MNRAS, 370, 468

Petit, P., Donati, J.-F., \& Collier Cameron, A. 2002, MNRAS, 334, 374

Petit, P., Dintrans, B., Morgenthaler, A., Van Grootel, V., Morin, J., Lanoux, J., Aurière, M., \& Konstantinova-Antova, R. 2009, A\&A, 508, p. L9

Skilling, J. \& Bryan, R. K. 1984, MNRAS, 211, 111 